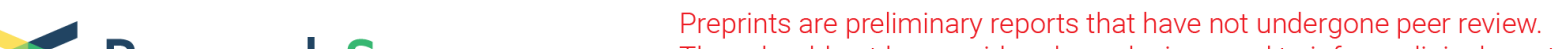 Research Square
or referenced by the media as validated information.
}

\section{Use Of Psychoactive Substances And Sociodemographics Characteristics In A Hospitalized Population With Mental Disorders In A Mid-Income Country}

JAIME CARMONA-HUERTA ( $\square$ jaime.carmona@academicos.udg.mx )

Instituto Jalisciense de Salud Mental https://orcid.org/0000-0002-0695-5452

Santiago Castiello-de Obeso

University of Oxford

Andrea Aguirre-Ramírez

Instituto Jalisciense de Salud Mental

Sara Pérez-Muñoz

Instituto Jalisciense de Salud Mental

David Cardona-Müller

Universidad de Guadalajara Centro universitario de ciencias de la Salud

Fernando Grover-Páez

Universidad de Guadalajara Centro universitario de ciencias de la salud

Bertha Iñiguez-Carvajal

Instituto Jalisciense de Salud Mental

Jesus Alejandro Aldana-López

Instituto Jalisciense de Salud Mental

Israel Itzaman-Jiménez

Instituto Jalisciense de Salud Mental

Rafael Medina-Dávalos

Instituto Jalisciense de Salud Mental

Research

Keywords: Drug addiction, drug abuse, ASSIST, methamphetamine, cannabis, ethanol, dual pathology, psychotic substance-induced disorder, substance use disorder

Posted Date: June 18th, 2020

DOI: https://doi.org/10.21203/rs.3.rs-34957/v1 
License: (c) (i) This work is licensed under a Creative Commons Attribution 4.0 International License. Read Full License 


\section{Abstract}

\section{Introduction:}

The use of psychoactive substances in patients diagnosed with mental illness is an increasing public health problem; the purpose of this work was to carry out a study to identify the most prevalent psychoactive drug abuse patterns in hospitalized patients in a psychiatric hospital, to estimate the severity of substance abuse, as well as to determine the factors associated with drug consumption using multivariate statistical modeling.

\section{Methods}

An analytical, retrospective study was carried out through the review of patient records discharged during 2018. Sociodemographic data, diagnoses, clinical follow-up information, clinimetric score of the alcohol consumption, Tobacco and Substances Detection Test (ASSIST 2.0) were obtained as well as the toxicological profile results.

\section{Results}

A representative sample of 268 participants was obtained, $61.6 \%$ men and $38.4 \%$ women, $86.6 \%$ unemployed, $67.9 \%$ single, with an average age of 36 years and 8 years of schooling. The most frequent diagnoses were Schizophrenia (42\%), Substance-Induced Psychotic Disorder (22\%), and Bipolar Disorder (17.9\%). The most frequent substances detected in urine toxicology test were: Cannabis $21.8 \%(n=45)$ and methamphetamines $10.7 \%(n=22)$. The substances with the most frequent consumption in the last 3 months were tobacco, ethanol, cannabis, and methamphetamines. The highest score in the severity of consumption were methamphetamines and cannabis. The most important predictors for ethanol consumption were tobacco and cannabis abuse, for cannabis abuse were schooling years and ethanol consumption, and for methamphetamine consumption, the most important predictors were cocaine and cannabis abuse.

\section{Conclusions}

Young, male, unemployed subjects, with lower educational level, a psychiatric condition, and psychoactive consumption represent an identifiable "sociodemographic phenotype" that has an increased risk of requiring hospital services on a more recurrent basis and that could represent a burden to the health system. Methamphetamines abuse continues to increase and is becoming a significant mental health and public health issue. Cannabis and methamphetamine use are more severe and have more serious repercussions in terms of psychiatric morbidity. Correct public policies must be designed to intervene in this population. 


\section{Introduction}

The use of psychoactive substances in patients diagnosed with mental illness is a growing public health problem (1). In general, patients with psychiatric disorders have a higher incidence of suffering a substance use disturbance compared to the general population (2). The disorders that present greater comorbidity with substance use disorders are mood, anxiety, psychotic disorders, and antisocial personality disorder (1) (3).

According to some studies, the lifetime prevalence of a substance use disorder in non-hospitalized people with a mental disorder is between 20 to $50 \%(1,3,4)$. In turn, the prevalence of some mental disorders in people admitted to specialized centers for substance abuse ranges from 50 to $75 \%$ of individuals $(3,5)$. Chan et al. (2008) found that young adult patients (18-25 years old) who were in residential treatment for substance use, were the most vulnerable to present psychiatric comorbidity, finding a prevalence between $42 \%-61 \%(6)$.

Regarding the comorbidity of substance use before admission in hospitalized psychiatric patients, studies have shown that the rates of such comorbidity are varied. In the United Kingdom, a study carried out in 2000 reported that $49 \%$ of hospitalized patients with functional psychosis had substance abuse or dependence (7). In the United States, it is estimated that almost a third of hospital admissions for adults with a primary psychiatric disorder have a concomitant substance use disorder (8), while in a study conducted in a psychiatric hospital in the Western Cape Province in South Africa, 23\% of patients met the criteria for substance abuse. The main substances of abuse were cannabis (27\%), alcohol (23\%), and methamphetamines (13\%) (9).

In low- and middle-income countries, studies related to substance use have mainly focused on the prevalence and correlation of substance use with mental health problems in the general population (10). In a study carried out in Colombia, a dual diagnosis prevalence of $7.2 \%$ was found, while in India prevalence of $32.4 \%$ was reported in the general population $(11,12)$. However, there are no studies on the use of substances in psychiatrically hospitalized patients and there are no publications where explanatory models are developed that describe the influence of the interaction of the different variables that characterize this phenomenon. For this reason, the purpose of this work was to carry out a study to (1) identify the most prevalent psychoactive drug abuse patterns in hospitalized patients in a psychiatric

hospital, (2) estimate the severity of substance abuse, and (3) determine the factors associated with drug consumption using multivariate statistical modeling.

\section{Material And Methods}

Study design and subjects

A review of the records of all the patients who were admitted in 2018 to the hospital "Prolonged stay center for comprehensive care in mental health" (CAISAME), the largest psychiatric hospital in western Mexico, was conducted. A total of 252 beds available for the hospitalization area. The site serves as a 
place of concentration for mental illnesses since it is the reference hospital for chronic or serious disorders in a network of 10 first-level mental health units distributed in a geographical area of the country (with a surface similar to the area that covers the country of Portugal and 3 times the size of the US state of Massachusetts) and that offers mental health care services for a population of approximately 8 to 10 million inhabitants (13).

The review began with the total number of patients who were discharged from January 1,2018 , to December 31 of the same year $(\mathrm{N}=712)$. A probabilistic and representative sample was obtained from this total when applying the formula to estimate the prevalence of substance use. The following parameters were taken into account: a) the total number of admissions in a year $(\mathrm{N}=712), \mathrm{b})$ maximum acceptable error $=5 \%, c$ ) estimated percentage of the sample $=50 \%, d$ ) desired level of confidence $=95 \%$ obtaining a sample number of 250 . It was decided to expand the sample by $6 \%$ if there were incomplete data in the review of records, so the final sample included 268 patients.

This review previously included the generation of a special format called the PCSPsi format (pattern of consumption of psychoactive substances) created from this project and which is part of the information gathering and evaluation program, to assess the quality of care, as well as for the development of research that favors improvements for said attention. The information included in the PCSPsi format was organized in 6 sections: sociodemographic data, diagnoses, clinical follow-up information, clinimetric score, the pattern of substance use, and results of toxicological profile. Through this format, the information was extracted to be placed in the database used for this investigation.

All information extracted from the files, captured in the PCSPsi format and later exported to the database, was converted into information that could not be related to any patient, hence, all subjects remained anonymous.

The present work is a retrospective and observational analytical type study; complied with the amendments established in the Helsinki declaration, safeguarding at all times the principles of beneficence, non-maleficence, autonomy, and justice; It was approved by the research department of the Jalisciense Institute of Mental Health with the registration number 2019-195.

\section{Procedures}

Six treating doctors were trained to fill out the PCSPsi format that included the Alcohol, Smoking, and Substance Involvement Screening Test (ASSIST) (see properties of the test in the next section), after that, another 3 health workers were in charge of extracting the information from that formats and establish the database. Neither the treating physicians who completed the forms nor the health workers in charge to extract the information obtained in the database were related to the content analysis, thereby maintaining the anonymity and blinding of the patients' results.

The filling of the PCSPsi forms was carried out systematically, it was not allowed to let any item unregistered since it could not be saved electronically if left empty. Section 1 collected sociodemographic 
information and included: age, sex, marital status, years of schooling, occupation, place of residence, practicing religion. Section 2 was concerned with diagnoses and included the registration of up to 3 psychiatric diagnoses and medical comorbidities. Section 3 contained the clinical follow-up information and included the record of the history of having already had "some previous treatment for any addiction", time of illness in years, time of treatment in years, previous hospitalizations, hospitalizations in the last 12 months, days of hospitalization in the present admission and the number of drugs prescribed at discharge. Section 4 contained the scores of the scales used in the project and included the Global Assessment of Functioning score (GAF) at admission and the GAF score at discharge, ASSIST scores for the consumption of ethanol, cocaine, cannabis, hallucinogens, methamphetamines, inhalants, opioids, sedatives/hypnotics/anxiolytics, tobacco, and other substances. Section 5 was made up of the variables of "substance use", the use of substances was recorded in the last 3 months, the severity of use according to the Diagnostic and Statistical Manual of Mental disorders Fifth Edition (DSM 5) and ASSIST for the clinical association of the main diagnosis with the use of substances. Finally, Sect. 6 was the one that included the results of the toxicological profile obtained from the urine sample within the first 24 hours of admission and included the analysis of cannabis, cocaine, methamphetamines, benzodiazepines, and opioids use.

\section{Measurements and categorizations}

The ASSIST scores were used to assess substance use. This questionnaire aims to identify levels of harmful use of substances. It consists of 8 items on the recent use (last 3 months) and throughout the life of 10 different substances (tobacco, alcohol, cannabis, cocaine, methamphetamines, or other stimulants, anxiolytics, hallucinogens, inhalants, and other drugs) (14). It has a sensitivity and specificity for detection of consumption of 0.80 / 0.71 for tobacco, 0.83 / 0.79 for alcohol, 0.910 .90 for cannabis, 0.92 / 0.94 for cocaine, 0.97 / 0.87 for methamphetamines, 0.94 / 0.91 for sedatives, and 0.94 / 0.96 for opioids (15).

"Substance use" was defined as the presence of consumption of any legal or illegal substance for nontherapeutic purposes in the degree of recreation, abuse, dependence or to mitigate deleterious symptoms that occur in the absence of such substance (withdrawal). It was defined as "a history of treatment for addictions" to the presence in their medical history of a therapeutic approach with the specific purpose of reducing or avoiding the consumption of some substance; "Active substance use" to any subject who has had a substance use in the last 3 months; "Pattern of light substance use", thus categorized through the ASSIST scores obtained ( 0 to 10 points for ethanol consumption and 0 to 3 points for the rest of the substances); "Pattern of moderate consumption of substances" thus categorized through the ASSIST scores obtained ( 11 to 26 points in ethanol consumption and 4 to 26 points for the rest of substances); "Pattern of severe substance use", thus categorized through the ASSIST scores obtained (27 or more points in the use of any substance); "Disease time in years", period of time since the main diagnosis for which the patient was admitted to the hospital in this occasion, that is, his main diagnosis and until the date of his admission to it; "Time of disease treated in years" to the period of time elapsed from the 
beginning of a therapeutic approach for its main diagnosis and until this hospitalization; "Number of drugs on discharge" to the last pharmacological prescription indicated on the day of hospital discharge.

For the description of the main psychiatric diagnoses and medical comorbidities, the international classification of diseases version 10 (ICD 10) was used.

The sociodemographic variables described were sex, marital status, occupation, place of residence, and schooling.

The clinical variables described were: age in years, time of the disease in years, time of the disease that has been treated in years, number of hospitalizations in the last 12 months, number of hospitalizations in their entire lives, days of hospital stay, prescribed psychotropic drugs at discharge, GAF score on admission, GAF score on discharge, substance use at some time in life, substance use in the last 3 months and severity of use.

\section{Statistical analysis}

The sociodemographic data and the results of the nominal and ordinal variables (diagnoses, comorbidities, substance use, and pharmacological groups used) were presented through frequencies and percentages; the results of continuous variables (age, schooling in years, number of hospitalizations, days of hospital stay, clinimetric test score, prescribed drugs, and doses) are shown as a mean and standard deviation. A probability of type 1 or Alpha error of 0.05 was considered.

When comparing two means, the student's t-test was used for independent samples, however, if the criteria for normality and homoscedasticity were not met, the Mann Whitney $\mathrm{U}$ test was used.

Furthermore, we perform a predictive analysis for the 3 dependent variables (DV) that were considered the most relevant (1. Use of alcohol, 2. Use of cannabis, and 3. Use of methamphetamines). In a first moment, we fitted three multivariate logistics models (one for each DV), and in a second stage, we apply a backward stepwise algorithm to reduce the irrelevant factors of the models. The independent variables (IV) or regressors that we used if appropriate were: consumption in the last 3 months of (1) methamphetamines, (2) cocaine, (3) cannabis, (4) alcohol, (5) hallucinogens, (6) inhalants, (7) opioids, (8), Sedatives, Hypnotics and Anxiolytics (SHA), (9) tobacco, and (10) others, (11) treatment time, (12) days of hospital stay, (13) sex, (14) age, (15) diagnosis, (16) years of education. See the exact models in the supplementary material. (The code is available upon request from the authors). Of course, when predicting a DV like the use of alcohol, we did not include this use of alcohol as an IV, the same for the use of cannabis and the use of methamphetamines models.

For each DV, complete models were created, which were called "full model" (Mfull), which included the interactions of the most relevant variables (methamphetamines, alcohol, and cannabis) with the rest of the regressors. We used the "Mfull" as the input for a backward stepwise regression using Akaike Information Criterion (AIC). The algorithm consists of a method of eliminating the factors that provide 
less predictive information to the model (we use the stepAIC function from the Modern Applied Statistics with S package; Venables, \& Ripley, 2002). In this case a factor $i$ is removed to create a "reduced model" (Mred), which will be compared with "Mfull"; and if the AIC of Mred < Mfull then factor $i$ will be removed. The previous step is repeated until the AIC of Mred > Mfull. To see the result, step by step of the 3 models, see Supplementary Materials.

The final models reduced after the stepwise algorithm were called "final model", (Mfinal). For all the 3 Mfinal coefficients, the following were obtained: standard error, T value, standardized coefficient, standardized coefficient error, p-value, effect size, and $95 \%$ confidence interval; the McFadden approximation was obtained and the Hosmer and Lemeshow goodness of fit test was carried out. The alpha value of 0.05 was used.

We used the statistical package for the social sciences SPSS version 25 and "RStudio" which uses the R language developed and updated by "The R Project for Statistical Computing". The statistical packages used in R were: for data processing, reshape2 and dplyr $(16,17)$; McFadden R2 approximations for logistic models, pscl (18); Hosmer and Lemeshow test (19); model writing, psycho (20); and data visualization, ggplot2 (21), viridis (22), and ggpubr (23).

\section{Results}

Use of psychoactive substances and sociodemographic characteristics

268 subjects were included in the study, $61.6 \%$ were male, the mean age was 36 years, an average of 8 years of schooling, $86.6 \%$ were unemployed, $67.9 \%$ were single, with an average 9 years with the main psychiatric diagnosis and 3.4 hospitalizations throughout their lives; the detailed description of the characteristics of the population included is reported in Tables 1 and 2. 
Table 1

Sociodemographic variables. $(n=268)$

\begin{tabular}{|c|c|c|}
\hline Variables & Categories & Frequency \% \\
\hline \multirow[t]{2}{*}{ Sex } & Male & $165(61.6)$ \\
\hline & Female & $103(38.4)$ \\
\hline \multirow[t]{4}{*}{ Marital status } & Single & $182(67.9)$ \\
\hline & Married/Free union & $50(18.7)$ \\
\hline & Divorced/separated & $26(9.7)$ \\
\hline & Widower & $10(3.7)$ \\
\hline \multirow[t]{2}{*}{ Occupation } & Employed & $36(13.3)$ \\
\hline & Unemployed & $234(86.7)$ \\
\hline \multirow[t]{5}{*}{ Schooling } & No schooling & $21(7.8)$ \\
\hline & Elementary school & $106(39.6)$ \\
\hline & Middle school & $87(32.5)$ \\
\hline & High school & $38(14.2)$ \\
\hline & Bachelor & $16(6.0)$ \\
\hline \multirow[t]{3}{*}{ Residency } & Metropolitan area & $167(62.3)$ \\
\hline & State resident (not from the metropolitan area) & $83(31.0)$ \\
\hline & Another state & $18(6.7)$ \\
\hline
\end{tabular}


Table 2

Continues variables $(n=268)$

\begin{tabular}{|l|l|}
\hline Variables & Mean (ED) \\
\hline Years of schooling & $8.0(3.7)$ \\
\hline Age & $36.5(12.1)$ \\
\hline Disease duration & $9.0(9.9)$ \\
\hline Treatment duration & $7.1(9.6)$ \\
\hline Hospitalizations in the last year & $1.3(0.8)$ \\
\hline Lifetime hospitalizations & $3.4(5.1)$ \\
\hline Hospitalization duration (days) & $28.2(22.9)$ \\
\hline Number of psych drugs prescribed on discharge & $2.2(0.9)$ \\
\hline GAF score (admission) & $17.1(5.9)$ \\
\hline GAF score (discharge) & $58.5(13.5)$ \\
\hline
\end{tabular}

The three most frequent discharge diagnoses were: Schizophrenia with $42.2 \%(n=113)$, substanceinduced psychotic disorder with $22.0 \%(n=59)$ and bipolar disorder with $17.9 \%(n=48)$, which together represent $82.1 \%$ of the sample. The remaining diagnoses were major depressive disorder $4.1 \%(n=11)$, schizoaffective disorders $2.2 \%(n=6)$, intellectual disability $1.1 \%(n=3)$, personality disorder $1.1 \%(n=3)$ and other diagnoses. $9.4 \%(n=25)$.

Substance abuse in the last 3 months was distributed as follows: $46.6 \%$ of the sample used tobacco, $45.9 \%$ ethanol, $34.3 \%$ cannabis, and $32.8 \%$ methamphetamines (Table 3 ).

Toxicological urine tests were carried out to detect consumption of some substances in $76.9 \%(n=206)$ of the total number of hospitalized patients within the first 24 hours of admission. The 4 substances that were detected most frequently were: Cannabis in $21.8 \%(n=45)$, methamphetamines in $10.7 \%(n=22)$, benzodiazepines in $5.3 \%(n=11)$ and cocaine in $0.5 \%(n=1)$ subjects. 
Table 3

Substance abuse in the last three months $(n=268)$

\begin{tabular}{|lll|}
\hline \multirow{2}{*}{ Substance } & \multicolumn{2}{l|}{ Frequency (\%) } \\
\cline { 2 - 3 } & Consumption & No consumption \\
\hline Tobacco & $125(46.6)$ & $143(53.4)$ \\
\hline Ethanol & $123(45.9)$ & $145(54.1)$ \\
\hline Methamphabis & $92(34.3)$ & $176(65.7)$ \\
\hline Cocaine & $88(32.8)$ & $180(67.2)$ \\
\hline Benzodiazepines & $19(7.1)$ & $249(92.9)$ \\
\hline Inhalants & $17(6.3)$ & $253(93.7)$ \\
\hline Hallucinogens & $2(0.7)$ & $266(99.3)$ \\
\hline Opioids & $1(0.4)$ & $269) 99.6)$ \\
\hline
\end{tabular}

The severity of substance abuse

The 4 substances that had the highest frequency of consumption in the last 3 months were tobacco, ethanol, cannabis, and methamphetamines. For tobacco consumption $(n=125)$, a pattern of mild consumption was observed in $14.4 \%$ of people, $71.2 \%$ moderate consumption and $14.4 \%$ severe consumption. For ethanol consumption $(n=123), 43.9 \%$ had a slight consumption, $43.1 \%$ a moderate and $13 \%$ a severe consumption. In cannabis users $(n=92) 10.9 \%$ had a light consumption, $45.7 \%$ a moderate consumption and $43.5 \%$ a severe consumption. Finally, for methamphetamine users $(n=88)$ there was a slight consumption in $5.7 \%$ people, moderate in $45.5 \%$ and severe in $48.9 \%$ (Fig. 1).

Regarding the severity of consumption, the 2 substances that obtained the highest score were methamphetamines and cannabis, since $16.0 \%$ and $14.9 \%$ of the total sample, respectively, obtained a score higher than the cut-off point (27 points) to classify the use of a substance as severe consumption (Fig. 1).

Multivariate Statistical Modeling

To explore the associated factors for drug consumption and to respond to our third objective, we carried out a predictive multivariable analysis. The next section presents the exploratory analysis used to identify the association between alcohol, cannabis and methamphetamines consumption with several independent variables (for details see Methods).

Alcohol abuse final logistical model 
In the overall Mfina/predicting model, alcohol use has an explanatory power of $73.72 \%$. The model's intercept is at $-3.17 \pm 0.83$. Within this model: The effect of tobacco use is significant (beta $=2.17 \pm 0.35$, $95 \% \mathrm{Cl}$ [1.49 to 2.87], $\mathrm{p}<.001$ ) and can be considered as large (std. beta $=2.17 \pm 0.35$ ). The effect of cannabis use is significant (beta $=1.30 \pm 0.42,95 \% \mathrm{Cl}[0.50$ to 2.13$], \mathrm{p}<.01$ ) and can be considered as medium (std. beta $=1.30 \pm 0.42$ ).

Cannabis abuse final logistic model

In The overall Mfina/predicting model cannabis use has an explanatory power of $85.75 \%$. The model's intercept is at $-1.22 \pm 1.07$. Within this model: The effect of schooling years is significant (beta $=-1.73 \pm$ $0.80,95 \% \mathrm{Cl}[-3.33$ to 0.16$], \mathrm{p}<.05)$ and can be considered as large (std. beta $=-1.73 \pm 0.80)$. The effect of alcohol use is significant (beta $=1.67 \pm 0.50,95 \% \mathrm{Cl}[0.70$ to 2.69$], \mathrm{p}<.001$ ) and can be considered as large (std. beta $=1.67 \pm 0.50$ ). The effect of substance induced psychotic disorder is significant (beta $=$ $1.52 \pm 0.54,95 \% \mathrm{Cl}[0.48$ to 2.62$], \mathrm{p}<.01$ ) and can be considered as large (std. beta $=1.52 \pm 0.54$ ). The effect of tobacco use is significant (beta $=1.45 \pm 0.48,95 \% \mathrm{Cl}$ [0.51 to 2.42], $\mathrm{p}<.01$ ) and can be considered as medium (std. beta $=1.45, \pm 0.48$ ). The effect of amphetamine use is significant (beta $=$ $1.35 \pm 0.45,95 \% \mathrm{Cl}[0.47$ to 2.26$], \mathrm{p}<.01$ ) and can be considered as medium (std. beta $=1.35 \pm 0.45$ ).

Methamphetamines abuse final logistical model

In the overall Mfina/predicting model, methamphetamines use has an explanatory power of $86.92 \%$. The model's intercept is at $-1.30 \pm 1.09$. Within this model: The effect of substance induced psychotic disorder is significant (beta $=2.33 \pm 0.52,95 \% \mathrm{Cl}$ [1.33 to 3.40], $\mathrm{p}<.001$ ) and can be considered as large (std. beta $=2.33 \pm 0.52$ ). The effect of cocaine use is significant (beta $=1.82 \pm 0.70,95 \% \mathrm{Cl}[0.53$ to 3.31], $p<.01$ ) and can be considered as large (std. beta $=1.82 \pm 0.70$ ). The effect of cannabis use is significant (beta $=$ $1.50 \pm 0.48,95 \% \mathrm{Cl}$ [0.56 to 2.47$], \mathrm{p}<.01$ ) and can be considered as large (std. beta $=1.50 \pm 0.48$ ). The effect of Tabaco is significant (beta $=1.25 \pm 0.59,95 \% \mathrm{Cl}[0.08$ to 2.43$], \mathrm{p}<.05$ ) and can be considered as medium (std. beta $=1.25 \pm 0.59$ ). The effect of age is significant (beta $=-0.05 \pm 0.02,95 \% \mathrm{Cl}[-0.09$ to $-0.01], \mathrm{p}<.01$ ) and can be considered as small (std. beta $=-0.63 \pm 0.24$ ).

The summary of the three final models used to predict methamphetamine, cannabis, and alcohol consumption are presented in Fig. 2.

\section{Discussion}

It is worth noting that the set of sociodemographic characteristics of the sample studied here appear to be associated with an increased risk for presenting mental disorders, since the average included subjects were young, male adults, who were single and unemployed. These characteristics have been associated with this increased risk in studies previously carried out and similar to ours $(24,25)$.

It is important to highlight the high percentage of unemployment that the participants had, some authors have observed an increase of 1.5 to 2 times more the risk of being hospitalized in unemployed or 
economically inactive subjects (26).

It is documented that people with severe mental illness, such as schizophrenia and bipolar disorder, have high unemployment rates, with more difficulties in finding and keeping a job, which may be due to low levels of education, stigma, and chronic or recurrence of symptoms (27). The more severe the mental disorder, the greater the association with unemployment or economic inactivity has been reported, as is the case of psychotic disorders (the most prevalent syndrome in the sample studied here), which are associated with less remunerated jobs and integrate the lowest socio-economic strata of a community (26).

In individuals with sociodemographic characteristics such as those observed here, there is usually an increase in the rate of psychiatric hospital readmissions. Bui and Wijesekera (2019) found a statistically significant association between the unemployment rate of the population and the rate of hospital admissions, this association was closer in case of also having a substance abuse disorder (28).

According to a recent study carried in South Africa on people with mental illness who were hospitalized, unemployment is often more common in patients with a psychotic disorder. This may be due to the deterioration that accompanies severe and persistent mental disorders, such as schizophrenia, and that leads to conditions of inequality that prevent them from carrying out the fair competition in the labor market (29). A study carried out in Colombia with people who had been admitted to a psychiatric hospital reported similar results to those found in our study; the sample was made up of single young men, of low socioeconomic strata, in them, $62.6 \%$ had ages between 18 and 34 years, with low levels of education, $49.5 \%$ presenting the diagnosis of schizophrenia or other disorders psychotic. They also reported high percentages of unemployment, however, they observed that this phenomenon was higher (51.6\%) among those with a dual diagnosis compared to those without substance abuse (45.5\%) (30). Specifically, there is consistent evidence of the relationship between the association of unemployment with substance abuse in young adults $(25,31,32)$. Besides, almost a third of patients with chronic psychotic disorders meet the criteria for dual illness, with a three-fold higher prevalence in men, in whom a lower age at their first hospitalization and a greater number of hospitalizations compared to people with similar characteristics, but without a history of consumption (33).

All this accounts for the implicit relationship between the variables unemployment, substance use, and severe and persistent mental disorder. This combination generates in the people who present it, an entity that has a different clinical impact and carries a higher risk of admission at younger ages, a greater number of readmissions, a reduction in quality of life, and greater dysfunction $(34,35)$.

On the other hand, there is a striking increase in the incidence of the diagnosis of "psychotic disorder induced by substance abuse" as the main admission diagnosis, since in previous studies in our environment this nosologic entity was not considered as one of the first 3 causes of admission. This reflects a negative repercussions that are beginning to appear in the social fabric of the region due to the influence of the overwhelming drug trafficking and consumption, thus reflecting the newer problems that our society is experiencing $(36,37)$.

Page $13 / 24$ 
According to the data obtained, the most commonly abused substances in the studied sample in the last 3 months were: tobacco (46.6\%), ethanol (45.9\%), cannabis (34.3\%) and methamphetamines (32.8\%), similar to reports in the study carried out by Rodriguez and Colls in Spain, where tobacco, ethanol, and cannabis were the substances with the highest use before hospitalization for mental illness, differing only by the fourth substance in frequency, where cocaine replaced Methamphetamines (38).

Regarding tobacco (the most used substance by the patients in this study), its relevance lies in the fact that it exerts a negative effect among the patients under treatment with antipsychotics, since the components of the tobacco are known to be potent inducers of the enzymatic cytochrome P450 complex, in particular, the $1 \mathrm{~A} 2$ isoform, increasing the rate of biotransformation of drugs that are metabolized by this enzyme. This increased metabolism of some antipsychotics (such as olanzapine and clozapine) results in a reduction in their plasma concentration, which may complicate the evolution of their pathology, even leading to the current relapse responsible for their latest hospitalization (39).

Regarding ethanol, it is known that its abuse precedes the consumption of other substances, finding ethanol in the category of "entry drug" (40). Previous studies show the existence of a clearly defined sequence of drug abuse that begins with licit substances and leads to illicit drugs, with ethanol being the most common entry substance. It is worth considering then that future interventions should be directed at alcohol abuse prevention, management, and rehabilitation (41-47).

Cannabis use (34.3\%) was in third place among the most commonly abused substances in the last 3 months and first place among illegal substances. It also ranked second (14.9\%) in terms of severity of use, second only to methamphetamine (16\%). That is, cannabis and methamphetamines not only have a high prevalence in terms of their abuse, but they were the 2 substances that were used most dangerously, according to the ASSIST scores. Regarding the use of cannabis and its association with psychosis, it has been observed that patients with psychotic disorders have a higher prevalence of cannabis use and consumption disorder compared to the general population. Besides, compared to those who do not consume this substance, cannabis abuse increases the risk of developing Psychosis, severe and continuous consumption increases the risk of developing schizophrenia. There is also a dose-dependent association, with an increased risk of psychosis in frequent users (48). Consumption is also associated with earlier onset of psychotic disorders, occurring on average 2.7 years earlier than in people who do not consume it (48). Cannabis users with psychotic disorders are at increased risk of relapse,

hospitalizations, poorer adherence to treatment, and greater severity of positive symptoms. Discontinuing use reduces the risk of progressing to a poor prognosis (48).

Just as there is a "sociodemographic phenotype" for the entire sample, the specific sociodemographic characteristics of methamphetamine users are also relevant. They are the youngest group of people compared to the rest of the sample, with the shortest disease evolution, the fewest number of hospitalizations, a higher GAF score at discharge. Except for hallucinogens and opioids abuse, methamphetamine abuse was associated with the greatest severity in consumption (according to the ASSIST scores). They are a group with great potential for a timely intervention prior to their evolution 
towards a "revolving door" phenomenon that is difficult to manage. Given that, in their last hospitalization, their stay did not last for more days compared to the rest of the sample, did not have a greater number of prescribed psychotropic drugs, they entered with similar severity, but were discharged with greater recovery. This evidenced by the GAF score at both times $(49,50)$. It would then be necessary to devise public policies or a specific management plan to avoid relapses in these patients upon discharge (51).

In Mexico, Medina-Mora et al. reported that the consumption of methamphetamine ranks third of those substances for which treatment is sought (14.3\%), however, in regions near the border, it occupies the first place of attention (31.2\%) (37). In the present study, the consumption of methamphetamines ranked fourth, with $32.8 \%$, among the most used substances in the last 3 months. Of the patients who consume, the majority (48.9\%) report severe consumption. According to reports from the Inter-American Drug Abuse Control Commission in 2019, Mexico ranks second in prevalence, in the general population, of methamphetamine use with $0.9 \%$, being higher in men (52). However, the source of monitoring is based on population-based surveys, which have limitations that reduce their usefulness for evaluating shortterm changes, decrease the possibility of obtaining representative figures for specific vulnerable groups, and have limitations regarding the validity of the information derived from the social stigma surrounding consumption, so these figures may be underestimated (53).

The risks in the consumption of this substance directly impact the public health of the country to a greater degree than the consumption of other substances, its use usually increases by 3 or 3 fold the risk of presenting psychotic symptoms, particularly if it is used in large quantities or an early age onset (54.55). Methamphetamines cause an overflow of dopamine in the striatum, leading to excessive glutamate release in the cortex. Excess glutamate can, over time, damage cortical interneurons. This damage causes dysregulation of the thalamocortical signal, which can result in the presentation of psychotic symptoms (55).

Regarding predictive analysis, the logistic models for methamphetamines, ethanol, and cannabis had a high power to explain the totality of the consumption phenomenon $(86.92 \%, 73.72 \%$, and $85.75 \%$ respectively). In other words, each of the variables that made up the models, by being present together with the other variables, can predict with significant statistical power the possibility that in a similar scenario there may or may not be a risk of consuming any of these substances.

It is interesting to observe how the findings in our study and others suggest that the risk of illicit substance consumption considered as "more dangerous" is increased if other substances for legal consumption were previously used, or if 2,3 or more substances were added before the arrival of cocaine or methamphetamine use. It is of utmost importance to highlight that the sociodemographic variable with the highest weight in these models was the years of study, with an effect size of -1.73 , which shows that at a lower school level there is a greater risk of cannabis use, with the consequent risk that this entails for being one of the "entry drugs" for the consumption of other illicit drugs. It is also relevant to emphasize that at a younger age the risk of consumption of other substances increases, with a particularly large 
effect size (from - 0.63) being observed for the use of methamphetamines, in the presence of consumption of other substances (alcohol, cannabis or cocaine).

As part of the limitations of the study, we found a high percentage of people who did not undergo the toxicological test in the first 24 hours of admission $(23.1 \%$ did not have this test, mainly due to the level of agitation reported by nursing), which reduced the detection figures for acute substance use. Besides, the fact that the methamphetamine detection test has a reduced positive predictive value in terms of its completion time concerning the date of last use (detects a positive use only if it was performed within 48 to 72 hours ) further reduced the percentage of people identified as consumers of this substance in the last 12 weeks (56).

However, we consider that the most important limitation of our work is the predictive level that the results show us, since being an exploratory and observational study, the evidence presented here only allows to establish possible associations and suggestions for future studies to be carried out to determine if the sociodemographic and clinical characteristics mentioned here as "risk variables" could be replicated in prospective studies designed to obtain a better quality of evidence.

\section{Conclusions}

We consider that it is vitally important to identify the characteristics and profiles of the population admitted to psychiatric institutions. It is crucial to investigate the factors that modulate the clinical course of mental illness over time. For example, the traits that differentiate the different consumption patterns of psychoactive substances. The results obtained in our study corroborate the data of international studies on the significant influence of substance use in the population with mental disorders. Especially in those with more severe conditions requiring hospital admission. Also, it allows us to establish a sociodemographic profile of the people who represent a high burden on mental health care systems. Young men, unemployed, less educated, with a psychiatric condition and psychoactive drug consumption represent an identifiable "sociodemographic phenotype" that will require hospital services on a more recurring basis.

For us, the recent positioning of drug use like methamphetamines and its impact on mental health was highly striking. The proof is the modification of the morbidity profiles of this psychiatric institution, where psychotic disorders induced by psychoactive substances already displaced bipolar diseases that traditionally occupied second place after schizophrenia. Although substances such as tobacco and alcohol continue to be more prevalent, the present study showed that the patterns of illegal psychoactive drugs such as cannabis and methamphetamines are especially more severe and have more serious repercussions in terms of psychiatric morbidity. The systematization of this type of study will be pending over time to identify changes and the repercussions that these could generate. Therefore, we consider that it is essential that low and middle-income countries generate this type of information for the design of correct public policies tailored to each of the countries or regions that generate them. 


\section{Abbreviations}

AIC: (Akaike Information Criterion) ASSIST: The Alcohol, Smoking and Substance Involvement Screening Test; CAISAME: Centre of Integral Care in Mental Health; Cl: Confidence Interval; DSM: Diagnostic and Statistical Manual of Mental Disorders; DV: Dependent Variables; GAF: Global Assessment of Functioning; ICD: International Statistical Classification of Diseases and Related Health Problems; IV: Independent Variables; MFINAL: Final Models; MFULL: Full Models; MRED: Reduced Models PCSPsi: Pattern of Consumption of Psychoactive Substances; SHA: Sedatives, Hypnotics and Anxiolytics; SPSS: Statistical Package for the Social Sciences; STD: standardized; US: United States

\section{Declarations}

\section{- Ethics approval and consent to participate:}

This retrospective study followed the recommendations and guidelines established in the Helsinki declaration and its four major principles: beneficence, nonmaleficence, justice, and autonomy. It was approved by the ethics and investigation committee of the Instituto Jalisciense de Salud Mental (Jalisco's Mental Health Institute) with the following number: 2019 - 195. The patients signed an informed consent form allowing the inclusion of their data in the present study.

\section{- Consent for publication:}

Not applicable

\section{- Availability of data and material:}

The datasets used and/or analyzed during the current study are available from the corresponding author on reasonable request.

\section{- Competing interests}

The authors declare that they have no competing interests.

\section{- Funding}

There was no funding for this work.

\section{- Acknowledgments}

We would like to thank the "Instituto Jalisciense de Salud Mental" and its director, Francisco Ramírez Barreto MD for their support in carrying out this work, as well as the University of Guadalajara for their 
contribution to the viability of this project.

- Authors' contributions

Conceptualization: JC, SC, RM. Data curation: SC, AA, SP, and BI. Formal analysis: JC, SC. Funding acquisition: JC, DC, and FG. Investigation: JC, SC, DC, FG, JA, II, and RM. Methodology: JC, SC, BI, and RM. Project administration: JC. Resources: JC, DC, FG, JA, II, BI, and RM. Software: JC and SC. Supervision: JC, JA, and RM. Validation: II, AA, and SP. Visualization: JC and SC. Writing - original draft: JC, SC, AA, SP, and RM. Writing - review \& editing: JC, JA, II, DC, FG, and RM. All authors read and approved the final manuscript.

\section{References}

1. Regier DA. Comorbidity of Mental Disorders with Alcohol and Other Drug Abuse. JAMA. 1990;264(19):2511-9. https://doi.org/10.1001/jama.1990.03450190043026.

2. Substance Abuse and Mental Health Services Administration. (2019). Key substance use and mental health indicators in the United States: Results from the 2018 National Survey on Drug Use and Health (HHS Publication No. PEP19-5068, NSDUH Series H-54). https://www.samhsa.gov/data.

3. Marín-Navarrete R, Benjet C, Borges G, Hernández AE, Nanni-Alvarado R, Ayala-Ledesma, FernandezMondragón J, Medina-Mora ME. Comorbilidad de los trastornos por consumo de sustancias con otros trastornos psiquiátricos en Centros Residenciales de Ayuda-Mutua para la Atención de las Adicciones. Salud Mental. 2013;36(6):471-9. https://doi.org/10.17711/sm.0185-3325.2013.057.

4. Ross S, Peselow E. Co-Occurring Psychotic and Addictive Disorders. Clin Neuropharmacol. 2012;35(5):235-43. https://doi.org/10.1097/wnf.0b013e318261e193.

5. Substance Abuse and Mental Health Services Administration (SAMH-SA) and Center for Substance Abuse Center (CSAT). (2005). Substance abuse treatment for persons with co-occurring disorders. Treatment improvement protocol Vol. 42. https://store.samhsa.gov/system/files/sma13-3992.pdf.

6. Chan Y, Dennis ML, Funk RR. Prevalence and comorbidity of major internalizing and externalizing problems among adolescents and adults presenting to substance abuse treatment. J Subst Abuse Treat. 2008;34(1):14-24. https://doi.org/10.1016/j.jsat.2006.12.031.

7. Phillips P, Johnson S. (2003). Drug and alcohol misuse among in-patients with psychotic illnesses in three inner-London psychiatric units. Psychiatr Bull, 27(6), 217-20. https://doi.org/10.1192/pb.27.6.2176.

8. Blader JC. Acute Inpatient Care for Psychiatric Disorders in the United States, 1996 Through 2007. Arch Gen Psychiatry. 2011;68(12):1276-83. https://doi.org/10.1001/archgenpsychiatry.2011.84.

9. Weich L, Pienaar W. Occurrence of comorbid substance use disorders among acute psychiatric inpatients at Stikland Hospital in the Western Cape, South Africa. African Journal of Psychiatry. 2009;12(3):213-7. https://doi.org/10.4314/ajpsy.v12i3.48496. 
10. Borges G, Benjet C, Orozco R, Medina-Mora ME. A longitudinal study of reciprocal risk between mental and substance use disorders among Mexican youth. J Psychiatr Res. 2018;105:45-53. https://doi.org/10.1016/j.jpsychires.2018.08.014.

11. Castaño Pérez GA, Sierra Hincapié GM. Trastorno dual en población general de Itagüí, Colombia. Revista Colombiana de Psiquiatría. 2016;45(2):108-17. https://doi.org/10.1016/j.rcp.2015.08.005.

12. Subodh BN, Hazari N, Elwadhi D, Basu D. Prevalence of dual diagnosis among clinic attending patients in a de-addiction center of a tertiary care hospital. Asian Journal of Psychiatry. 2017;25:169-74. https://doi.org/10.1016/j.ajp.2016.10.020.

13. Carmona-Huerta J, Castiello-de Obeso S, Ramírez-Palomino, Polypharmacy in a hospitalized psychiatric population: risk estimation and damage quantification. BMC Psychiatry. 2019, 21;19(1):78. http://doi.org10.1186/s12888-019-2056-0.

14. WHO ASSIST Working Group. The Alcohol, Smoking, and Substance Involvement Screening Test (ASSIST): development, reliability, and feasibility. Addiction. 2002;97(9):1183-94. https://doi.org/10.1046/j.1360-0443.2002.00185.x.

15. Humeniuk R, Ali R, Babor TF, Farrell M, Formigoni ML, Jittiwutikarn J, De Lacerda RB, Ling W, Marsden J, Monteiro M, Nhiwatiwa S, Pal H, Poznyak V, Simon S. Validation of the alcohol, smoking, and substance involvement screening test (ASSIST). Addiction. 2008;103(6):1039-47. https://doi.org/10.1111/j.1360-0443.2007.02114.x.

16. Wickham H. Reshaping Data with the reshape Package. J Stat Softw. 2007;21(12):1-20. http://www.jstatsoft.org/v21/i12/.

17. Wickham H, François R, Henry L, Müller K. (2019). dplyr: A Grammar of Data Manipulation. R package version 0.8.0.1. https://CRAN.R-project.org/package=dplyr.

18. Jackman S. (2017). pscl: Classes and Methods for R Developed in the Political Science Computational Laboratory. United States Studies Centre, University of Sydney. Sydney, New South Wales, Australia. R package version 1.5.2. https://github.com/atahk/pscl/.

19. Lele SR, Keim JL, Solymos P. (2019). Resource selection: Resource Selection (Probability) Functions for Use-Availability Data. R package version 0.3-5. https://CRAN.Rproject.org/package=ResourceSelection.

20. Makowski D. (2018). The Psycho Package: An Efficient and Publishing-Oriented Workflow for Psychological Science. Journal of Open Source Software, 3(22), 470. Available from https://github.com/neuropsychology/psycho.R.

21. Wickham H. ggplot2: Elegant Graphics for Data Analysis. New York: Springer-Verlag; 2016.

22. Garnier S. (2018). viridis: Default Color Maps from 'matplotlib'. R package version 0.5.1. https://CRAN.R-project.org/package=viridis.

23. Kassambara A. (2018). ggpubr: 'ggplot2' Based Publication Ready Plots. R package version 0.2. https://CRAN.R-project.org/package=ggpubr.

24. Katz G, Durst R, Shufman E, Bar-Hamburger R, Grunhaus L. Substance Abuse in Hospitalized Psychiatric Patients. Israel Medical Association Journal. 2008;10:672-5. 
https://www.ima.org.il/FilesUploadPublic/IMAJ/0/45/22731.pdf.

25. Habibisaravi R, Navaeinia S, Farnia S, Zarghami M. Alcohol, Cannabinoids, and Opioids Abuse and Dependence Among Psychiatric Inpatients. Iranian Journal of Psychiatry Behavioral Sciences. 2015;9(1):1-4. https://doi.org/10.17795/ijpbs229.

26. Castelpietra G, Balestrieri M, Bovenzi M. Occupational status and hospitalisation for mental disorders: findings from Friuli Venezia Giulia region, Italy, 2008-2017. International Journal of Psychiatry in Clinical Practice. 2019;23(4):265-72. https://doi.org/10.1080/13651501.2019.1611864.

27. Modini M, Tan L, Brinchmann B, Wang M, Killackey E, Glozier N, Mykletun A, Harvey SB. Supported employment for people with severe mental illness: Systematic review and meta-analysis of the international evidence. Br J Psychiatry. 2016;209(1):14-22. https://doi.org/10.1192/bjp.bp.115.165092.

28. Bui TA, Wijesekera N. Unemployment and the rate of psychoactive-substance-related psychiatric hospital admission in regional Queensland: An observational, longitudinal study. Australasian Psychiatry. 2019;27(4):388-91. https://doi.org/10.1177/1039856219859265.

29. $10.1097 /$ MD.0000000000018399

Madala-Witbooi N, Adeniyi O. (2020). Demographic and clinical profiles of admitted psychiatric patients of the East London Mental Health Unit in the Eastern Cape, South Africa. Medicine 2019;98:52. http://dx.doi.org/10.1097/MD.0000000000018399.

30. Castaño Pérez GA, Rojas Bernal LA, Bareño Silva J, Sierra Hincapié GM. Sociodemographic and clinical factors associated with dual disorders in a psychiatric hospital. Salud mental. 2017;40(6):279-90. https://doi.org/10.17711/sm.0185-3325.2017.036.

31. Lee JO, Hill KG, Hartigan LA, Boden JM, Guttmannova K, Kosterman R, Bailey JA, Catalano RF. Unemployment and substance use problems among young adults: Does childhood low socioeconomic status exacerbate the effect? Soc Sci Med. 2015;143:36-44. https://doi.org/10.1016/j.socscimed.2015.08.016.

32. Catalano R, Goldman-Mellor S, Saxton K, Margerison-Zilko C, Subbaraman M, LeWinn K, Anderson E. The Health Effects of Economic Decline. Annu Rev Public Health. 2011;32(1):431-50. https://doi.org/10.1146/annurev-publhealth-031210-101146.

33. Florentin S, Rosca P, Raskin S, Bdolah-Abram T, Neumark Y. Psychiatric Hospitalizations of Chronic Psychotic Disorder Patients with and Without Dual Diagnosis, Israel, 1963-2016. Journal of Dual Diagnosis. 2019;15(3):130-9. https://doi.org/10.1080/15504263.2019.1609149.

34. Cotton S, Lambert M, Schimmelmann B, Foley D, Morley K, McGorry P, Conus P. Gender differences in premorbid, entry, treatment, and outcome characteristics in a treated epidemiological sample of 661 patients with first episode psychosis. Schizophr Res. 2009;114(1-3):17-24. https://doi.org/10.1016/j.schres.2009.07.002.

35. Marquez-Arrico J, Río-Martínez L, Navarro J, Prat G, Forero D, Adan A. Coping Strategies in Male Patients under Treatment for Substance Use Disorders and/or Severe Mental Illness: Influence in 
Clinical Course at One-Year Follow-Up. Journal of Clinical Medicine. 2019;8(11):1-15. https://doi.org/10.3390/jcm8111972.

36. Benassini O. La atención psiquiátrica en México hacia el siglo XXI. Salud Mental. 2001;24(6):62-73. https://www.redalyc.org/pdf/582/58262408.pdf.

37. Medina-Mora ME, Real T. The Drug Scene in Mexico and the Road Ahead. Adicciones. 2013;25(4):294-9. https://doi.org/10.20882/adicciones.29.

38. Rodriguez R, Aragues M, Jimenez MA, Ponce G, Muñoz A, Bagney A, Joenicka J, Palomo T. Dual diagnosis in psychiatric inpatients: prevalence and general characteristics. Investigación Clínica. 2008;49(2):195-205. https://www.ncbi.nlm.nih.gov/pubmed/18717266.

39. Taylor D, Paton C, Kapur S. The Maudsley Prescribing Guidelines in Psychiatry. 12th ed. Hoboken: Wiley-Blackwell; 2015.

40. Kleinig J. Ready for Retirement: The Gateway Drug Hypothesis. Substance Use Misuse. 2015;50(89):971-5. https://doi.org/10.3109/10826084.2015.1007679.

41. Kandel DB, Yamaguchi K, Chen K. Stages of progression in drug involvement from adolescence to adulthood: further evidence for the gateway theory. J Stud Alcohol. 1992;53(5):447-57. https://doi.org/10.15288/jsa.1992.53.447.

42. Stawicki S, Jordan A, Salen P, Wojda T, Cohen M, Hasani A, Luster J, Seoane J, Stankewicz H. Exploring the association between initial serum alcohol concentration and polysubstance use: More than a simple "gateway drug" effect? International Journal of Critical Illness Injury Science. 2018;8(4):201-6. https://doi.org/10.4103/ijciis.ijciis_65_18.

43. Kirby T, Barry AE. Alcohol as a Gateway Drug: A Study of US 12th Graders. J Sch Health. 2012;82(8):371-9. https://doi.org/10.1111/j.1746-1561.2012.00712.x.

44. Barry AE, King J, Sears C, Harville C, Bondoc I, Joseph K. Prioritizing Alcohol Prevention: Establishing Alcohol as the Gateway Drug and Linking Age of First Drink With Illicit Drug Use. J Sch Health. 2015;86(1):31-8. https://doi.org/10.1111/josh.12351.

45. Witkiewitz K, Litten RZ, Leggio L. Advances in the science and treatment of alcohol use disorder. Science Advances. 2019;5(9):1-11. https://doi.org/10.1126/sciadv.aax4043.

46. Gustafson DH, McTavish FM, Chih M, Atwood AK, Johnson RA, Boyle MG, Levy MS, Driscoll H, Chisholm SM, Dillenburg L, Isham A, Shah D. A Smartphone Application to Support Recovery From Alcoholism. JAMA Psychiatry. 2014;71(5):566-72. https://doi.org/10.1001/jamapsychiatry.2013.4642.

47. Akbar M, Egli M, Cho Y, Song B, Noronha A. Medications for alcohol use disorders: An overview. Pharmacol Ther. 2018;185:64-85. https://doi.org/10.1016/j.pharmthera.2017.11.007.

48. Hasan A, von Keller R, Friemel C, Hall W, Schneider M, Koethe D, Leweke F, Strube W, Hoch E. Cannabis use and psychosis: a review of reviews. Eur Arch Psychiatry Clin Neurosci. 2019. https://doi.org/10.1007/s00406-019-01068-z.

49. Hajebi A, Amini H, Kashani L, Sharifi V. Twelve-month course and outcome of methamphetamineinduced psychosis compared with first episode primary psychotic disorders. Early Intervention in 
Psychiatry. 2016. https://doi.org/10.1111/eip.12404.

50. Kittirattanapaiboon P, Mahatnirunkul S, Booncharoen H, Thummawomg P, Dumrongchai U, Chutha W. (2010). Long-term outcomes in methamphetamine psychosis patients after first hospitalisation. Drug Alcohol Review, 29(4), 456-61. https://doi. org/10.1111/j.1465-3362.2010.00196.x.

51. Ma J, Li X, Wang T, Li S, Meng S, Blow F, et al. Relationship between the duration of methamphetamine use and psychotic symptoms: A two-year prospective cohort study. Drug Alcohol Dependence. 2018;187:363-9. https://doi.org/10.1016/j.drugalcdep.2018.03.023.

52. Hynes, M., Clarke, P., Cumsille, F., Araneda-Ferrer, J., Ahumada, G. (2019). The Report on Drug Use in the Americas 2019. 246-248. http://www.cicad.oas.org

53. Cruz-Cruz C, Vidaña-Pérez D, Mondragón y Kalb M, Martínez-Ruiz MJ, Olaiz-Fernández G, HernándezLezama LF, Hernández-Ávila M, Barrientos-Gutiérrez T. Medición de drogas ilícitas en aguas residuales: estudio piloto en México. Salud Pública de México. 2019;61(4):461-9. https://doi.org/10.21149/9819.

54. Salani D, Valdes B, De Santis J, Zdanowicz M. Back With a Vengeance: The Reappearance of Methamphetamine and its Implications for Health Care Providers. The Journal For Nurse Practitioners. 2020. https://doi.org/10.1016/j.nurpra.2020.03.005.

55. Hsieh J, Stein D, Howells F. The neurobiology of methamphetamine induced psychosis. Frontiers in Human Neuroscience. 2014;8:537, 1-12. 10.3389/fnhum.2014.00537.

56. 10.1016/j.mayocp.2016.12.007 Moeller KE, Kissack JC, Atayee RS, Lee KC. (2017). Clinical Interpretation of Urine Drug Tests. Mayo Clinic Proceedings, 92(5), 774-796. https://doi.org/10.1016/j.mayocp.2016.12.007.

\section{Figures}




\section{Consumption severity}

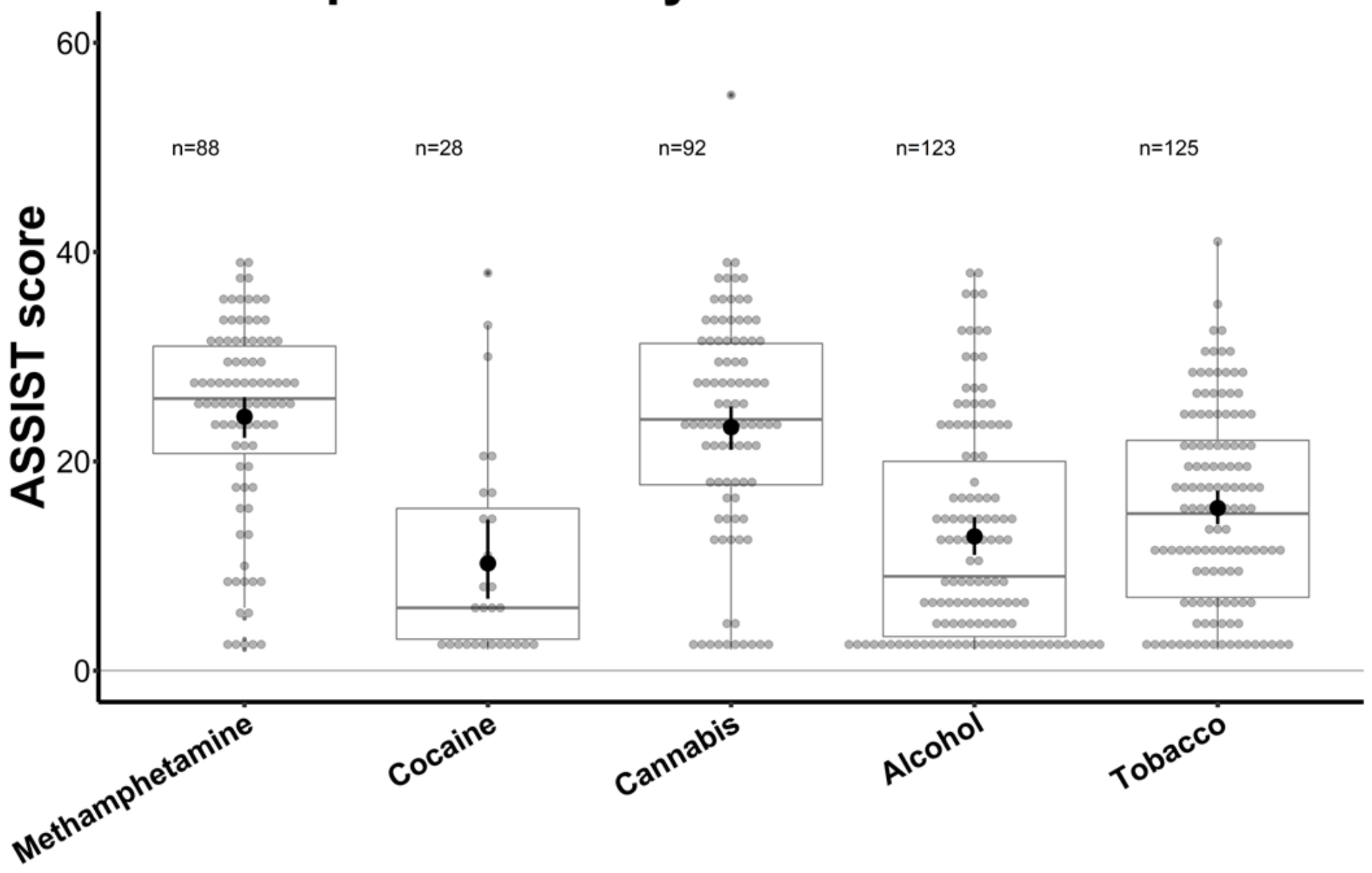

Figure 1

ASSIST scores on the severity of consumption per interest drugs. Each point is a patient. The black dot is the mean and the error bars are standard errors. 
Final models significant factors

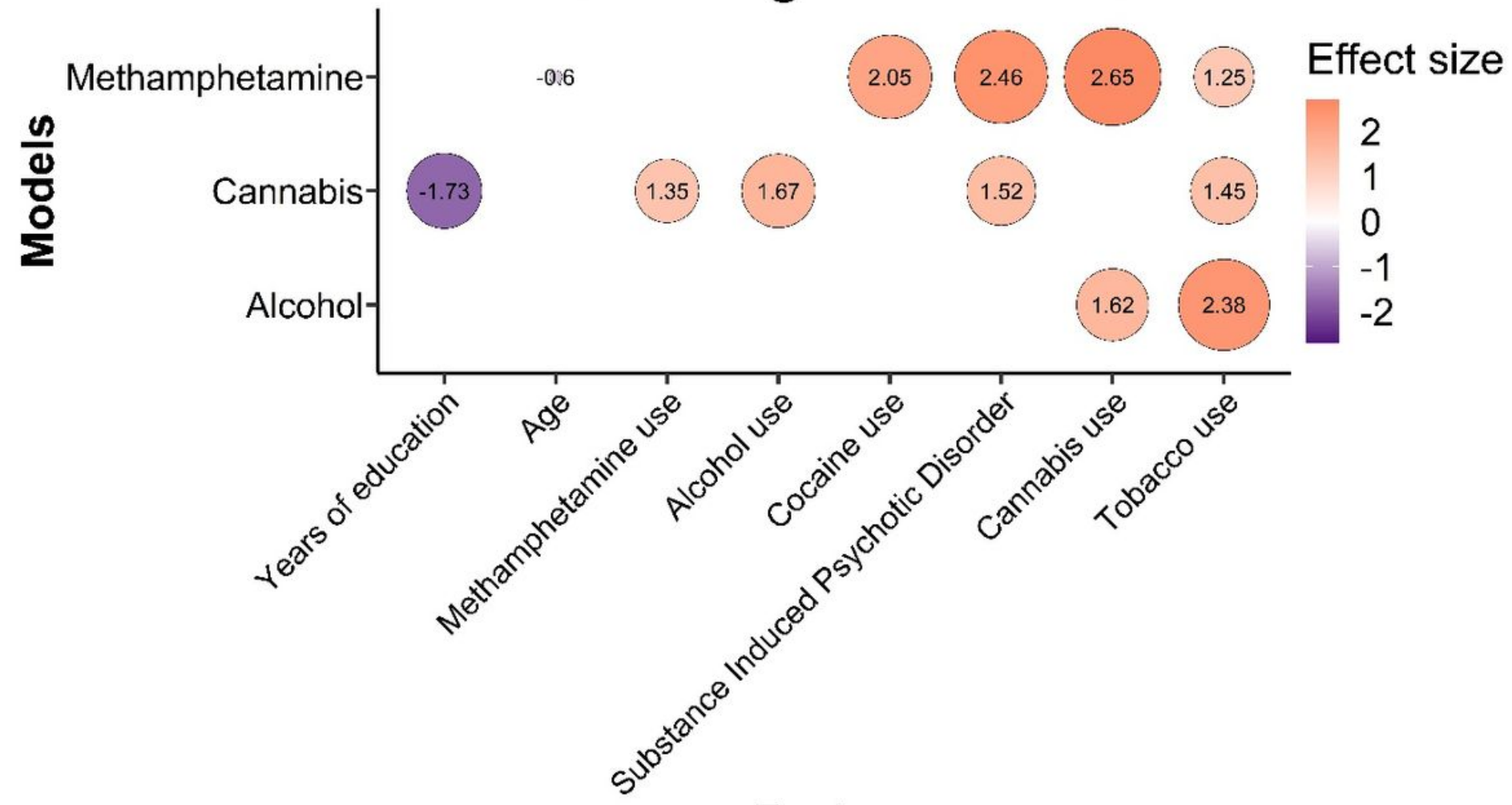

Factors

Figure 2

In this figure, we synthesis the 3 final logistic models: (1) methamphetamines use, (2) cannabis use, and (3) alcohol use in the last 3 months. The $y$-axis represents the 3 models and $x$-axis the regressors (or predictive factors) that survive the stepwise model reduction algorithm. When there are not purple and orange circles, it means that the model does not have that factor as a predictor. The circle size represents the effect size of a predictor, and the color is the direction of the effect. 